\title{
Role of CD73 and extracellular adenosine in disease
}

\author{
Presented by Maria P. Abbracchio
}

\author{
Simon C. Robson
}

Published online: 4 November 2011

(C) Springer Science+Business Media B.V. 2011

\section{Introductory Note}

Two important papers on the role of CD73, one of the enzymes involved in adenine nucleotide metabolism, have recently been published in two high level impact journals: The New England Journal of Medicine and The Journal of Clinical Investigation. One of these article deals with the involvement of CD73 in vascular calcification and the other one with the potential role in tumor growth. These new studies have great relevance for the development of novel therapeutic approaches to cardiovascular diseases and cancer. The highlights of these articles follow where these new findings are extensively and critically discussed.

\section{Highlight N. 1: Extracellular adenosine and CD73 in vascular disease}

Elliot Tapper ${ }^{1}$, Moritz Schmelzle ${ }^{1}$, Holger K. Eltzschig ${ }^{2}$ and Simon C. Robson ${ }^{1}$

${ }^{1}$ Beth Israel Deaconess Medical Center, Harvard University, Boston MA, USA

${ }^{2}$ University of Colorado, Aurora, CO, USA

Correspondence: srobson@bidmc.harvard.edu

St. Hilaire C, Ziegler SG, Markello TC, et al. (2011) "NT5E mutations and arterial calcifications". N Engl J Med 2011;364:432-42

S. C. Robson $(\bowtie)$

Beth Israel Deaconess Medical Center, Harvard University, Boston, MA, USA

e-mail: srobson@bidmc.harvard.edu

\section{Article summary}

This landmark manuscript by St. Hillaire et al implicates CD73 in adult onset vascular calcification [1]. St. Hillaire and colleagues present translational work that identifies hitherto unknown recessive mutations in NT5E amongst patients with a shared phenotype, namely delayed vascular calcification. In a series of elegant experiments, they demonstrate a functional defect in CD73, recapitulate that defect by transferring the mutation in NT5E to previously unaffected cells and then repair the functional defect using both genetic rescue and by circumventing the pathway with exogenous adenosine.

Vascular calcification is known to be associated with an excess risk of cardiovascular events and has been linked to common, clinically important diseases such as diabetes and renal disease [2]. The role of purinergic signalling in the pathogenesis of vascular calcification was first suggested by the study of idiopathic infantile arterial calcification, an autosomal recessive disease caused by a loss-offunction mutation in the ectonucleotide pyrophosphatasephosphodiesterase 1 gene (ENPP1), which generates pyrophosphate and AMP from ATP [3]. The published case series by St. Hillaire et al identifies nine persons from three families with calcifications of lower-extremity arteries and hand and foot joint capsules [1]. Each related a medical history and examination consistent with reduced peripheral blood flow manifested as pain with activity, also known as claudication. Imaging techniques confirmed the widespread, circumferential deposition of calcium along the (primarily infrarenal) peripheral arterial tree. Genomic analysis yielded a series of NT5E mutations each of which resulted in non-functional CD73. For example, the five affected members of family 1 shared a single 22.4-Mb region of homozygosity on chromosome 6 and had 
a homozygous nonsense mutation $(\mathrm{c} .662 \mathrm{C} \rightarrow \mathrm{A}, \mathrm{p} . \mathrm{S} 221 \mathrm{X}$ ) in NT5E. Family 2's affected members were homozygous for a missense mutation (p.C358Y) and the lone affected member of family 3 had one copy of NT5E with a frameshifted premature stop codon and one copy of p.C358Y. None of the heterozygous family members share the phenotype of vascular calcification. Cultured fibroblasts from affected members of "Family 1" show markedly reduced expression of NT5E messenger RNA, CD73 protein and enzyme activity, as well as increased alkaline phosphatase levels and accumulated calcium phosphate crystals. Genetic-type "rescue" experiments normalized the CD73 and alkaline phosphatase activity in patients' cells, and adenosine treatment decreased the levels of alkaline phosphatase and calcification.

\section{Commentary}

There are other purinergic-type ectoenzymes, which are expressed by the vasculature. Among these, CD39, the product of ectonucleoside triphosphate diphosphohydrolase 1 (ENTPD1) is responsible for the conversion of ATP to ADP and AMP (whereas NT5E encodes CD73, which converts AMP to adenosine). Adenosinergic/purinergic signalling along the arterial tree, modulated by CD39 and CD73, has been implicated in vascular injury [4-6]. ADP mediated platelet activation plays a central role in thrombogenesis and vascular inflammation and blocking this effect has saved countless lives [7]. Indeed, myocardial infarction and stroke, the condicio sine qua non of vascular injury, are both prevented and treated using irreversible P2Y12 inhibitors, namely clopidogrel and, recently, prasugrel $[8,9]$. Vascular injury of this sort is considered a consequence of unfettered inflammation, which, in turn, has been implicated in the pathogenesis of diabetes, renal disease, atheroscleosis and vascular calcification [10].

\section{Important insights, possible caveats and future directions for research}

Research on adenosinergic mechanisms of inflammation is growing and as it is found to be of importance in such interconnected, perhaps mutually dependent conditions, it helps unify the clinical picture. Take, for example, ENTPD-1 deletion (i.e. CD39 null), this results in hepatic insulin resistance in murine models [11] and CD39 expression has been shown to correlate with fasting plasma glucose and haemoglobin A1C levels in humans. [12] Similarly, ENTPD-1 impacts diabetic nephropathy in murine models and functional polymorphisms of the gene also appear to influence renal outcomes in African Americans with diabetes [13-15].
Against the backdrop of burgeoning indirect evidence, this work by St. Hillaire et al indicates that purinergic mechanisms directly impact vascular endothelial health. Yet, vascular calcification is a common lesion downstream of separate but convergent disease processes. It is important to recognize that while vascular calcifications can arise from single gene mutations encoding critical elements of purine metabolism - as they have in these rare, isolated cases - they arise in common diseases, such as diabetes and renal disease, which, while certainly multifactorial, possibly share purinergic mechanisms underpining the pathophysiology. St. Hillaire and colleagues have proposed that decreased adenosine generation secondary to failure of ecto-5 -nucleotidase expression on vascular cells, which in turn increases tissue nonspecific alkaline phosphatase (TNAP). Increased TNAP activity could certainly clear pyrophosphate generated by ENPP1 and might mimic outcomes of the more severe form of vascular calcification noted in generalized arterial calcification of infancy; the latter characterized by mutations in ENPP1 and low generation rates of pyrophosphate ab initio [3]. Although deletion of Enpp1 in mice models disease in infancy with soft tissue and arterial medial calcification associated with ectopic osteochondral differentiation [16-18], it seems that $\operatorname{Cd} 73$ deletion in mice does not result in similar phenotypes [16, 18-20].

The authors are to be congratulated on this important clinical discovery. However, it is also feasible that other factors are at play given the delayed manifestations of clinical vascular disease in middle age, as observed here in NT5E deficiency.

First, we infer that the dominant ectonucleotidase in both vascular endothelium and smooth muscle is CD39 $[6,21,22]$. CD39 would generate free phosphate and nucleoside phosphates, unlike the ENPP1 ectoenzyme, and not produce pyrophosphate per se. Hence, using fibroblasts with typically limited CD39 expression to study impact of NT5E deletion on calcification as done by St. Hilaire et al, although understandable, may not be the appropriate system to study vascular injury.

Secondly, it is feasible that pyrophosphate might only be a substantive product of extracellular nucleotides in those vascular tissues where CD39 bioactivity is altered as in atherosclerosis [23], sepsis [24], transplant chronic rejection [25] or in the setting of genetic polymorphisms with altered ecto-enzymatic activity [14]. Such a scenario with altered CD39 bioactivity might explain the unusual distribution of arterial calcification seen in NT5E deficiency seen in this highly interesting publication.

Third, loss of CD39 bioactivity with accumulation of ATP and other nucleoside triphosphates may be expected to 
result in local 'feed-forward inhibition' of NT5E [26]. This interaction has the potential to cause secondary inhibition of adenosine generation with associated arterial calcification as noted here, even in individuals with potentially normal genetic studies.

Finally, we note one conundrum with the proposed model linking adenosinergic effects to TNAP. While physiologic levels of pyrophosphate may inhibit generation of hydroxyapatite crystal, this metabolite may also serve as a reservoir for generation of phosphate with pro-calcification potential. Therefore, it is feasible that excessive concentrations of extracellular pyrophosphate, mediated in part by upregulated ENPP1 expression might even stimulate vascular calcification, as noted in cartilage models [17]. Curiously, levels of ENPP1 appear low in calcified atherosclerotic plaque [27].

Clearly, this publication raises many interesting issues and provides important insights for further study.

\section{Highlight N. 2: Extracellular adenosine in cancer}

Moritz Schmelzle, Elliot Tapper and Simon C. Robson Beth Israel Deaconess Medical Center, Harvard University, Boston MA, USA

Correspondence: srobson@bidmc.harvard.edu

\section{Wang L, Fan J, Thompson LF, Zhang Y, Shin T, Curiel TJ, Zhang B. (2011) "CD73 has distinct roles in nonhematopoietic and hematopoietic cells to promote tumor growth in mice”. J Clin Invest 121(6):2371-82.}

\section{Article summary}

Here, Wang et al. have shown that purinergic mechanisms are intimately involved in the regulation of tumor growth [28]. The authors show a survival benefit for $C d 73$ null mice bearing transplanted tumors, when compared to wild type mice. Based on previous experiments, showing that deletion of $C d 73$ on tumor cells significantly improves anti-tumor T cell immunity [29], they have also challenged Cd73 null mice with CD73siRNA or control siRNA transfected tumor cells. Optimal anti-tumor effect is clearly achieved in $C d 73$ null mice transplanted with CD73siRNA transfected tumors.

Experiments with bone marrow chimeric mice (wild type in wild type, wild type in $C d 73$, and vice versa) reveal that CD73 expression patterns on both hematopoietic and non-hematopoietic cells contribute to the control of tumor growth. CD8+ cells are found in tumors at 12 days after tumor inoculation at higher numbers in $C d 73$ null mice, when compared to wild type mice. Experiments using deleting antibodies indicate that the survival benefit of $C d 73$ null mice is $\mathrm{CD} 8+$ cell dependent (rather than CD4+, NK or B cells). These data suggest the central importance of CD73-mediated hydrolysis of extracellular AMP to adenosine on transplanted tumor cells and by recipient cells.

The authors also study the role of CD73 in adhesion and migration in homing of $\mathrm{T}$ cells to tumors, in vitro and in vivo. Exposure of endothelial cells to 5 -adenosine monophosphate (5-AMP) or adenosine-5 N-ethylcarboxamide (NECA) attenuates $\mathrm{T}$ cell adhesion, whereas adenosine $5^{\prime}-(\alpha, \beta$-methylene)diphosphate (APCP) restored this. Transmigration of $\mathrm{T}$ cells across endothelial cells was shown to be impacted by CD73-mediated hydrolysis of extracellular AMP to adenosine in vitro. Experiments using specific and non-specific A2 receptor agonists suggest that $\mathrm{T}$ cell homing is largely mediated by A2B-type receptors. The authors further examine the role of CD73 on modulating tumor infiltating regulatory $\mathrm{T}$ cells (tumor Tregs) interactions with effector $\mathrm{T}$ cells. Importantly, $C d 73$ null effector $\mathrm{T}$ cells did not show any changes in mediating anti-tumor responses. CD4+ tumor Tregs directly inhibited $\mathrm{CD} 8+\mathrm{T}$ cell functions, such as interferon-gamma (IFN- $\gamma$ ) production, and consequently $\mathrm{CD} 8+\mathrm{T}$ cell-mediated anti-tumor effects in a CD73-dependent manner. As expected, adoptive transfer of wild type Tregs, but not Cd73 null Tregs, abrogate the tumor escape mechanisms seen in $C d 73$ null mice.

\section{Commentary}

This study suggests well-coordinated immune purinergic responses to tumor progression. Most importantly, this study shows that both local and systemic production of adenosine by CD73 (in tandem with CD39) might modulate anti-tumor responses in vivo.

These novel data suggest that both cancer cells and host-derived cells cooperatively mediate tumor immune evasion, and that this occurs in an CD73-dependent manner.

\section{Important insights, possible caveats and future directions for research}

In this work, bone marow-derived cells, inclusive of CD4+CD25+ Tregs, are shown to suppress systemic T cell expansion and effector functions. In contrast, tumor resident cells (TEC), e.g. endothelial cells, impact $\mathrm{T}$ cell functions locally by limiting their homing to tumors. Curiously, bone marrow derived cell compartments also contain CD39+ and CD73+ mesenchymal stromal cells (MSC). Immunomodulatory properties of MSCs might be also mediated through adenosine signaling [30]. Further, although controversial, in the setting of inflammation endothelial cells (EC) at defined 
vascular beds may arise from hematopoietic progenitor cells [31]. See Fig. 1 for schema.

Several papers have been recently published indicating that scavenging of extracellular ATP and the production of adenosine have direct effects on tumor growth and spread of metastases $[32,33]$. Functionally active CD39 and $\mathrm{CD} 73$ are expressed by regulatory types of immune cells, e.g. Tregs [34]. Recent papers have shown that Tregs promote metastatic tumor growth in a manner dependent upon the expression of $\mathrm{CD} 39$ and the generation of adenosine [32].

Here, the authors clearly show that CD73 expression inhibits anti-tumor immune responses. Homing was also shown to be dependent on A2B receptor pathways, albeit whether or not through cAMP-dependent pathways remains uncertain at this time [28]. The witnessed effects could be related to adenosinergic/purinergic modulation of leukocyte homing or possibly even to induction of apoptosis. ATP effects via P2X7 death receptor activation; as well as adenosine have been associated with apoptosis. Specifically, A2A receptor stimulation which inhibits the activation of virtually all tested functions of T-cells can further induce apoptosis in thymocytes. In an environment where there is a lot of cell turnover and death (as in tumors), adenosine may induce such effects. This apoptotic pathway is coupled to adenylate cyclase activation, induction of the regulatory Nur77 transcription factor, and the pro-apoptotic Bim [35]. CD73 deletion (as would CD39 deletion) might be conceived of as "leukocyte protective" by limiting the generation of fluxes of extracellular adenosine levels in much the same way as adenosine deaminase activity might.

Adenosinergic effects on leukocyte extravasation, Tregmediated supression of antitumor $\mathrm{T}$ cell expansion and effector functions are already established. However, this outstanding paper contains elegant experimentation and provides important insights into the biology of CD73 in cancer. Crucially, this work [28] is also further validated and confirmed by Stagg et al. [36]. It appears from both studies that CD73 may be targeted on tumor cells, Tregs and potentially nonhematopoietic cells in the promotion of immune responsiveness.

In conclusion, these recent publications dealing with CD73 raise many interesting issues and provide important insights into the development of future adjunct therapies in cancer. Generation of adenosine by CD73 (together with CD39) results in decreased effector functions of CD8 T cells

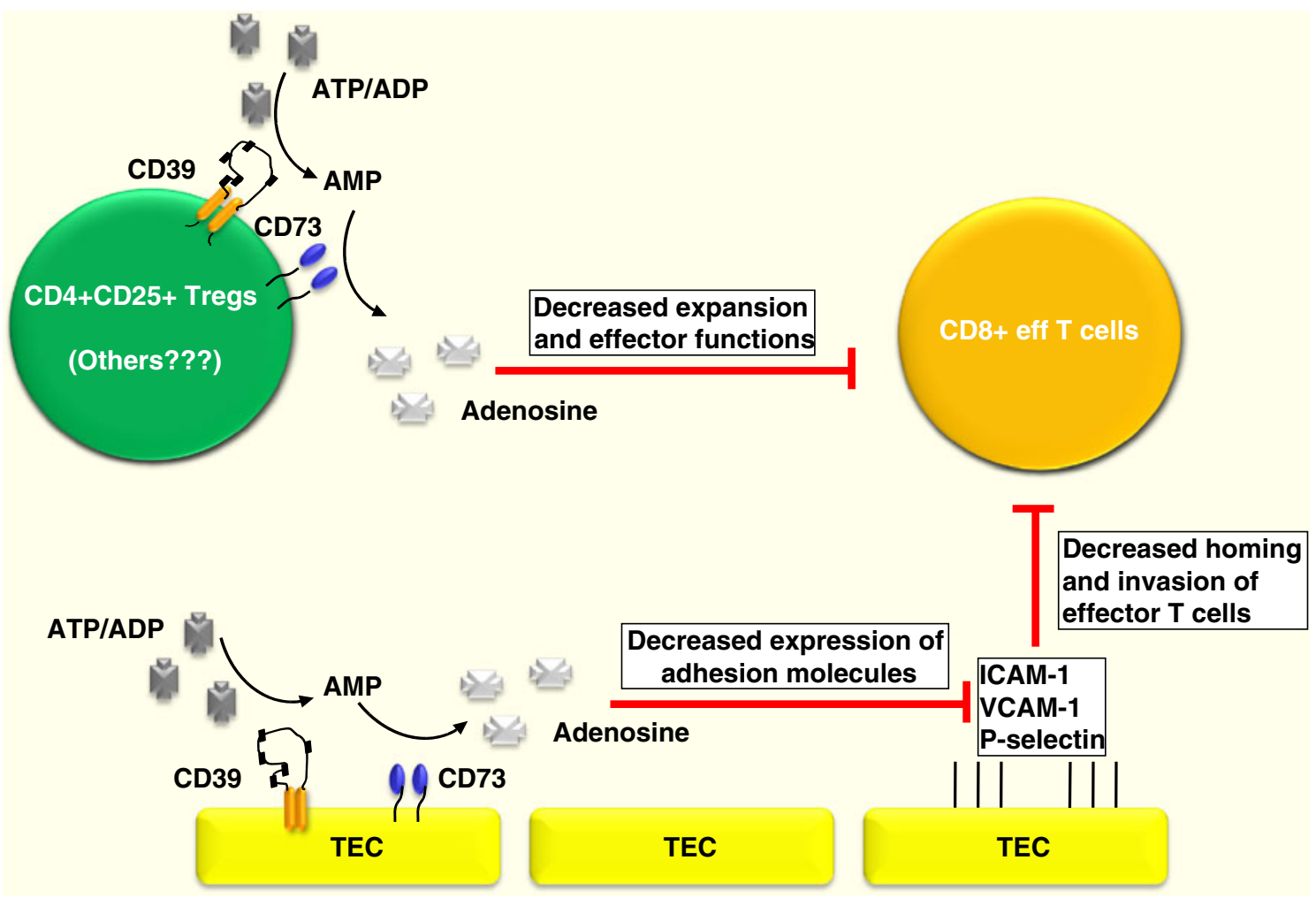

Fig. 1 Scheme of purinergic signaling pathways in cancer 
as well as limiting their homing to tumors by decreasing expression of adhesion molecules (see text for details).

\section{References}

1. St. Hilaire C, Ziegler SG, Markello TC, et al. NT5E mutations and arterial calcifications. N Engl J Med 2011;364:432-42.

2. Lehto S, Niskanen L, Suhonen M et al (1996) Medial artery calcification: a neglected harbinger of cardiovascular complications in non-insulindependent diabetes mellitus. Arterioscler Thromb Vasc Biol 16:978-83

3. Rutsch F, Ruf N, Vaingankar S et al (2003) Mutations in ENPP1 are associated with 'idiopathic' infantile arterial calcification. Nat Genet 34:379-81

4. Zernecke A, Bidzhekov K, Özüyaman B et al (2006) CD73/ Ecto-5-Nucleotidase Protects Against Vascular Inflammation and Neointima Formation. Circulation 113:2120-2127

5. Yang D, Zhang Y, Nguyen HG et al (2006) The A2B adenosine receptor protects against inflammation and excessive vascular adhesion. J Clin Invest 116:1913-1923

6. Kaczmarek E, Koziak K, Sévigny J et al (1996) Identification and characterization of CD39/vascular ATP diphosphohydrolase. J Biol Chem 271(51):33116-22

7. Solum NO (1999) Procoagulant expression in platelets and defects leading to clinical disorders. Arterioscler Thromb Vasc Biol 19:2841-2846

8. CAPRIE Steering Committee (1996) A randomised, blinded, trial of clopidogrel versus aspirin in patients at risk of ischaemic events (CAPRIE). Lancet 348(9038):1329-1339

9. Sabatine MS, Cannon CP, Gibson CM et al (2005) Addition of Clopidogrel to Aspirin and Fibrinolytic Therapy for Myocardial Infarction with ST-Segment Elevation. N Engl J Med 352:11791189

10. Haffner SM (2006) The Metabolic Syndrome: Inflammation, Diabetes Mellitus, and Cardiovascular Disease. Am J Cardiol 97 (suppl):3A-11A

11. Enjyoji K, Kotani K, Thukral C et al (2008) Deletion of cd39/entpd1 results in hepatic insulin resistance. Diabetes 57(9):2311-20

12. García-Hernández MH, Portales-Cervantes L, Cortez-Espinosa $\mathrm{N}$ et al (2011) Expression and function of $\mathrm{P} 2 \mathrm{X}(7)$ receptor and CD39/Entpd1 in patients with type 2 diabetes and their association with biochemical parameters. Cell Immunol 269 (2): $135-43$

13. Friedman DJ, Rennke HG, Csizmadia E et al (2007) The Vascular Ectonucleotidase ENTPD1 Is a Novel Renoprotective Factor in Diabetic Nephropathy. Diabetes 56:2371-2379

14. Friedman DJ, Talbert ME, Bowden DW et al (2009) Functional ENTPD1 Polymorphisms in African Americans With Diabetes and End-Stage Renal Disease. Diabetes 58:999-1006

15. Shirley DG, Vekaria RM, Sévigny J (2009) Ectonucleotidases in the kidney. Purinergic Signalling 5:501-511

16. Colgan SP, Eltzschig HK, Eckle T et al (2006) Physiological roles for 5'-ectonucleotidase (CD73). Purinergic Signal 2:35160

17. Rutsch F, Nitschke Y, Terkeltaub R (2011) Genetics in arterial calcification: pieces of a puzzle and cogs in a wheel. Circ Res 109 (5):578-92

18. Nitschke Y, Weissen-Plenz G, Terkeltaub R, Rutsch F. Npp1 promotes atherosclerosis in ApoE knockout mice. J Cell Mol Med. 2011 Apr 8. [Epub ahead of print]
19. Koszalka P, Ozüyaman B, Huo Y et al (2004) Targeted disruption of cd73/ecto-5'-nucleotidase alters thromboregulation and augments vascular inflammatory response. Circ Res 95:814-21

20. Thompson LF, Eltzschig HK, Ibla JC, Van De Wiele CJ, Resta R, Morote-Garcia JC, Colgan SP (2004) Crucial role for ecto-5'nucleotidase (CD73) in vascular leakage during hypoxia. J Exp Med 200(11):1395-405

21. Enjyoji K, Sévigny J, Lin Y et al (1999) Targeted disruption of cd39/ATP diphosphohydrolase results in disordered hemostasis and thromboregulation. Nat Med 5(9):1010-7

22. Robson SC, Sévigny J, Zimmermann H (2006) The ENTPDase family of ectonucleotidases: Structure function relationships and pathophysiological significance. Purinergic Signal 2(2):409-30

23. Lecka J, Bloch-Boguslawska E, Molski S, Komoszynski M (2010) Extracellular purine metabolism in blood vessels (Part II): Activity of ecto-enzymes in blood vessels of patients with abdominal aortic aneurysm. Clin Appl Thromb Hemost 16(6):650-7

24. Haskó G, Csóka B, Koscsó B, Chandra R, Pacher P, Thompson LF, Deitch EA, Spolarics Z, Virág L, Gergely P, Rolandelli RH, Németh ZH. Ecto-5'-Nucleotidase (CD73) Decreases Mortality and Organ Injury in Sepsis. J Immunol. 2011 Sep 14. [Epub ahead of print]

25. Robson SC, Wu Y, Sun X et al (2005) Ectonucleotidases of CD39 family modulate vascular inflammation and thrombosis in transplantation. Semin Thromb Hemost 31(2):217-33

26. Gordon EL, Pearson JD, Slakey LL (1986) The hydrolysis of extracellular adenine nucleotides by cultured endothelial cells from pig aorta. Feed-forward inhibition of adenosine production at the cell surface. J Biol Chem 261(33):15496507

27. Nitschke Y, Hartmann S, Torsello G, Horstmann R, Seifarth H, Weissen-Plenz G, Rutsch Fet al. Expression of NPP1 is regulated during atheromatous plaque calcification. J Cell Mol Med. 2009 Epub ahead of print] PMID:20015201

28. Wang L, Fan J, Thompson LF, Zhang Y, Shin T, Curiel TJ, Zhang B (2011) CD73 has distinct roles in nonhematopoietic and hematopoietic cells to promote tumor growth in mice. J Clin Invest 121(6):2371-82

29. Jin D et al (2010) CD73 on tumor cells impairs antitumor T-cell responses: a novel mechanism of tumor-induced immune suppression. Cancer Res 70(6):2245-2255

30. Saldanha-Araujo F et al (2011) Mesenchymal stromal cells up-regulate CD39 and increase adenosine production to suppress activated T-lymphocytes. Stem Cell Res 7(1):66-74

31. Harb R et al (2009) Bone marrow progenitor cells repair rat hepatic sinusoidal endothelial cells after liver injury. Gastroenterology 137 (2):704-12

32. Sun X et al (2010) CD39/ENTPD1 expression by CD4+FoxP3+ regulatory $\mathrm{T}$ cells promotes hepatic metastatic tumor growth in mice. Gastroenterology 139(3):1030-40

33. Feng $\mathrm{L}$ et al (2011) Vascular CD39/ENTPD1 directly promotes tumor cell growth by scavenging extracellular adenosine triphosphate. Neoplasia 13(3):206-16

34. Deaglio et al (2007) Adenosine generation catalyzed by CD39 and $\mathrm{CD} 73$ expressed on regulatory $\mathrm{T}$ cells mediates immune suppression. J Exp Med 204(6):1257-65

35. Kiss I et al (2006) Adenosine A2A receptor-mediated cell death of mouse thymocytes involves adenylate cyclase and Bim and is negatively regulated by Nur77. Eur J Immunol 36(6):1559-71

36. Stagg J et al (2011) CD73-deficient mice have increased antitumor immunity and are resistant to experimental metastasis. Cancer Res 71(8):2892-900 


\section{About the authors}

Moritz Schmelzle, M.D. is a research associate at the Transplant Institute, Division of Gastroenterology, Beth Israel Deaconess Medical Center, Harvard University, Boston, MA, USA. His research focuses on purinergic mechanisms in stem and immune cell responses in inflammation and organ restoration.

Elliot Tapper, M.D is a resident physician and research associate at Beth Israel Deaconess Medical Center, Harvard University,
Boston, MA, USA. His research focuses on purinergic mechanisms of nonalcoholic fatty liver disease and quality care in clinical hepatology.

Holger K. Eltzschig, M.D., Ph.D. is a physician scientist at the University of Colorado, Aurora, CO.

Simon C. Robson, M.D., Ph.D. is a physician scientist at Beth Israel Deaconess Medical Center, Harvard University, Boston, MA, USA 